\title{
Retrospective Review of Anesthetic Management of Free Flap Reconstructive Surgeries: An Analysis of 162 Cases
}

\author{
Harprit Kaur Madan ${ }^{1}$, Roshan Thawale ${ }^{2}$, Indrani Hemantkumar Chincholi ${ }^{3}$
}

\begin{abstract}
The success rate of free flap revascularization is affected by multiple factors. We did a retrospective study of 162 patients who underwent free flap reconstructive surgery over a period of approximately 4 years in the BYL Nair Hospital, a premier tertiary care public health center in Mumbai, Maharashtra, India. All the surgeries were performed by the same group of surgeons. A brief history of free flap surgery is presented, followed by a description of the anesthetic technique used and the results of the study. The various ways in which anesthetic management may influence the results of surgery are discussed. The data from 162 patients were collected and analyzed.

Keywords: Anesthesia, Free flap surgery, Microvascular circulation, Plastic surgery.

Research and Innovation in Anesthesia (2021): 10.5005/jp-journals-10049-0067
\end{abstract}

\section{INTRODUCTION}

Reconstructive free flap surgery is a complex method of wound closure for large wounds not amenable to linear (primary) closure. ${ }^{1}$

With the realization that the pedicle could be divided and the vessels reanastomosed at the recipient site, the field of free tissue transfer began to develop. In the mid-1950s, small bowel loops were used to replace the pharynx and the cervical esophagus. By the early 1970s, many workers were identifying islands of skin with a vascular pedicle that could be utilized for such tissue transfers. ${ }^{2}$

The procedure involves the transfer of free tissue (skin, muscle, bone, bowel, or a combination) to a site of tissue loss where its circulation is restored via microvascular anastomoses. A muscle flap produces a more even contour and better esthetic appearance than that achieved by a simple skin graft and provides a better defense against infection. A free flap has no lymph drainage, at least to begin with. It is denervated with respect to the sympathetic nervous system. It is usually only a single (and damaged) feeder artery and vein. ${ }^{1}$

The free flap is transferred with its accompanying artery and vein, which are then reattached to vessels at the donor site using microvascular techniques. ${ }^{3}$

The defect may have been caused by trauma, infection, or extensive surgery. Examples are:

- Trauma (e.g., fractures with overlying tissue loss, amputation)

- Burns

- Malignancy (e.g., head and neck cancers, malignant melanoma, ameloblastoma)

- Breast reconstructive surgery (e.g., transverse rectus abdominis muscle ['TRAM'] flap following mastectomy)

- Jaw reconstruction surgery postmandibulectomy.

The site and size of the defect determine which flap is used. The most commonly used flaps are the gracilis muscle for lower leg trauma, latissimus dorsi and rectus abdominis for breast reconstruction, pectoralis major and radial forearm flap for head and neck reconstruction, and free fibula graft for mandibular reconstruction. ${ }^{3}$
${ }^{1}$ Department of Anesthesiology, HBT Medical College and Dr RN Cooper Hospital, Mumbai, Maharashtra, India

${ }^{2}$ Department of Anesthesiology, Consultant in Pain Management and Anaesthetist Epsom and St Helier University Hospitals NHS Trust Carlshalton Surrey, UK

${ }^{3}$ Department of Anesthesiology, Seth GS Medical College and KEM Hospital, Mumbai, Maharashtra, India

Corresponding Author: Harprit Kaur Madan, Department of Anesthesiology, HBT Medical College and Dr RN Cooper Hospital, Mumbai, Maharashtra, India, Phone: +91 9870091313, e-mail: drpreet@hotmail.com

How to cite this article: Madan HK, Thawale R, Chincholi IH. Retrospective Review of Anesthetic Management of Free Flap Reconstructive Surgeries: An Analysis of 162 Cases. Res Inno in Anesth 2021;6(1):1-4.

Source of support: Nil

Conflict of interest: None

The stages of flap transfer are:

- Flap elevation and clamping of vessels

- Primary ischemia as blood flow ceases and intracellular metabolism becomes anaerobic (this is dependent on surgical time and lasts 60-90 minutes)

- Reperfusion as the arterial and venous anastomoses are completed and the clamps released

- Secondary ischemia is subsequent to hypoperfusion of the flap (minimized by appropriate anesthetic management). ${ }^{4}$

Despite improvements in surgical techniques, hypoperfusion and flap failure are still major concerns. Anesthesia can be an important factor in determining the success of this type of surgery through alterations in central hemodynamic, regional blood flow, and temperature regulation (Table 1).

\section{Materials and Methods}

This is a retrospective review of 162 patients who underwent freeflap reconstructive surgeries over a period of four years. The patients 
Table 1: Factors decreasing blood flow in free flaps $s^{5-7}$

\begin{tabular}{ll}
\hline Arterial & Arterial thrombosis \\
Venous outflow & Arterial spasm \\
& Venous thrombosis \\
& Venous spasm \\
& Mechanical compression (e.g. \\
dressings) & Excessive use of crystalloids \\
Flap edema & Extreme haemodilution \\
& Prolonged ischemia \\
& Histamine release (e.g. \\
& anesthetics, antibiotics) \\
& Excessive tissue handling \\
Gypovolemia & Hypothermia \\
& Pain \\
Respiratory alkalosis \\
Hypotension & Hypovolemia \\
& Cardiac depressant drugs (e.g. \\
anesthetics, calcium \\
channel blockers) \\
Extensive sympathetic blockade \\
(e.g. epidural) \\
Profound vasodilatation \\
Cardiac failure (e.g. ischemia, \\
fluid overload) \\
\\
\hline
\end{tabular}

belonged to ASA grades I, II, or III. All the free flaps reconstruction surgeries are included.

\section{Anesthetic Technique}

Although several anesthesiologists were involved over 4 years of this study, the anesthetic techniques employed were fundamentally the same for all cases reviewed. Preoperative assessment included elicitation of the patient's history to ascertain whether they had received chemotherapy which included bleomycin, which could result in gas exchange impairment as the drug is known to cause pulmonary fibrosis. Physical examination included assessment of mouth opening and upper airways to exclude potential intubation problems, and also inspection of peripheral veins, as many of these patients have received chemotherapy and multiple venesections leading to thrombosed veins. Other investigations included full blood count with clotting studies, assessment of blood urea nitrogen, serum creatinine, electrolytes, liver functions, ECG, and chest X-ray.

The patients were premedicated with opioids in combination with benzodiazepines. Anesthesia was induced via an indwelling needle in a peripheral vein with intravenously administered propofol $(2 \mathrm{mg} / \mathrm{kg})$, and then the patient was intubated orally or nasally depending on surgical site under a nondepolarizing relaxant, usually intravenous atracurium $(0.5-0.8 \mathrm{mg} / \mathrm{kg})$ or vecuronium (0.1-0.4 mg/kg). Cuffed endotracheal tubes were used orally and the nasal tubes were either reinforced or preformed. If difficulty with intubation was anticipated, the patient was anesthetized using an inhalation technique with sevoflurane and direct laryngoscopy attempted, and intubation was achieved with the assistance of either an introducer or a gum elastic bougie. The technique of blind nasal intubation was used earlier on, and fiberoptic bronchoscope was used more often during some of the more difficult intubations. A wide bore nasogastric feeding tube
Table 2: Postoperative care for free flap

\begin{tabular}{l}
\hline Postoperative care 7,8 \\
\hline Maintain normothermia \\
Hyperdynamic circulation_high cardiac output, low systemic \\
vascular resistance \\
Normal blood pressure \\
Haematocrit $30 \%$ (checked every 6 hours for the first 24 hours) \\
Urine output $>1 \mathrm{~mL} / \mathrm{kg} /$ hour \\
$\mathrm{SaO}_{2}>94 \%$ (supplemental oxygen for the first 24 hours) \\
Regular inspection of the flap and continuous monitoring of blood \\
flow in the flap by temperature and USG Doppler.
\end{tabular}

was usually passed after intubation, preferably to allow placement under direct vision. Venous access was provided by an 18 or $20 \mathrm{G}$ cannula in a peripheral vein and a central venous catheter inserted peripherally, whenever required. Access to jugular and subclavian veins was often restricted by the surgical field. A $20 \mathrm{G}$ cannula was placed in a radial artery in cases where major blood loss was anticipated. All patients had a urinary catheter placed.

The convective forced air warmer with blanket was used for temperature regulation, and anesthesia was maintained with nitrous oxide and oxygen with either isoflurane or sevoflurane. Analgesia was provided with either fentanyl or buprenorphine. Inspired gases were warmed and moistened by a condenser humidifier. Monitoring included electrocardiography, pulse oximetry, and also monitoring of inspired oxygen concentration, end tidal carbon dioxide, arterial blood pressure with waveform display, body temperature, neuromuscular blockade, bispectral index, and arterial blood pressure by indirect method. Intraoperatively, fluid maintenance was administered at a relatively high rate of 7-10 mL/ $\mathrm{kg} /$ hour of crystalloid, usually in the form of either lactated ringer or glucose $5 \%$ in normal saline $0.9 \%$. All infused solutions were warmed before infusion.

At the end of surgery, the neuromuscular blockade was reversed with neostigmine, along with glycopyrrolate or atropine and the patient was then transferred to the postanesthesia care unit, while breathing warmed, humidified oxygen-enriched air. An initial intravenous bolus of fentanyl $(0.5 \mu \mathrm{g} / \mathrm{kg})$ with or without midazolam was required to settle these patients, after which postoperative analgesia was generally provided by a fentanyl infusion ( $1 \mu \mathrm{g} / \mathrm{kg} / \mathrm{hour}$ ). All patients were given prophylactic intravenous antibiotics both pre- and postoperatively.

\section{Postoperative Management}

All these patients were managed postoperatively in high dependency unit setup ('PACU'). The patients were kept warm, pain-free, and well-hydrated.

Monitoring the flap: clinical observation of skin color, capillary return, temperature, and ultrasound Doppler were monitored for free skin flaps (Table 2).

\section{Results}

The age and sex distribution are shown in Table 3. There were 162 patients all falling into ASA classification 1-3, and mean age was 32.4, range 20-70 years. There were 103 males and 59 females.

Out of 162 patients, 5 patients required re-exploration surgeries. Three free flaps were salvaged out of 5 and 2 grafts completely failed (Table 4).

The mean perioperative blood loss was $1,100 \mathrm{~mL}$ with a range of $500-2,200 \mathrm{~mL}$, and mean perioperative blood replacement was 
Table 3: Age and sex distribution

\begin{tabular}{lccc}
\hline Age & Number & Male & Female \\
\hline $20-30$ & 54 & 22 & 32 \\
$30-40$ & 48 & 32 & 16 \\
$40-50$ & 36 & 29 & 7 \\
$50-60$ & 16 & 12 & 4 \\
$60-70$ & 8 & 8 & 0 \\
& 162 & 103 & 59 \\
\hline
\end{tabular}

Table 4: Procedure in which free flap transfer was performed

\begin{tabular}{lc}
\hline Surgery & Number \\
\hline Free scapular/parascapular flap for neck reconstruction & 8 \\
Free lateral arm flap for lip reconstruction & 4 \\
Free latissimus dorsi for sole reconstruction & 15 \\
Mandibulectomy with free fibular flap reconstruction & 50 \\
Breast reconstruction & 20 \\
Burns contracture release with free flap reconstruction & 20 \\
of joints & \\
Phallus repair with free radial artery flap & 2 \\
Free deep circumflex iliac artery flap with iliac bone for & 2 \\
maxillary and palate reconstruction & \\
Free deep inferior epigastric artery perforator flap for & 2 \\
breast reconstruction & \\
Toe transfer for thumb reconstruction & 2 \\
Double toe transfer for index and middle finger & 1 \\
reconstruction & \\
Anterio-lateral thigh free flap for postburn upper limb & 5 \\
reconstruction & \\
Anterio-lateral thigh free flap for oral malignancies & 4 \\
Anterio-lateral thigh free flap for maxillary & 7 \\
reconstruction & \\
Anterio-lateral thigh free flap for lower limb trauma & 5 \\
Free fibular flap for radial bone reconstruction & 3 \\
Free fibular flap for humerus bone reconstruction & 2 \\
Free fibular flap for palm reconstruction & 3 \\
Free radial artery flap for buccal carcinoma & 2 \\
Free radial artery flap for posttraumatic lip loss & \\
Free fibular flap for tibial bone reconstruction & 3 \\
\hline & 2 \\
\hline
\end{tabular}

1 unit with range of 1-3 units. Plasma expander in form of Dextran70 was given, mean volume $1,000 \mathrm{~mL}$ with range of $1,000 \mathrm{~mL}$ to $3,000 \mathrm{~mL}$. Mean urine output was $0.5-1 \mathrm{~mL}$ per $\mathrm{kg}$ body weight. Vasodilators were not used routinely, although 14 out of 162 cases (22.6\%) received small doses of esmolol (mean dose $10 \mathrm{mg} / \mathrm{kg}$ ), to reduce arterial pressure during the resection of the tumor mass, in order to minimize initial blood loss. 20 out of 162 (32.4\%) patients required postoperative ventilation, out of which 14 required only CPAP for 4-6 hours and were eventually extubated. Four of these 6 patients required pressure support ventilation for a period varying from 12 to 72 hours and were extubated (Table 4).

Postoperative analgesia was provided by continuous intravenous opiate infusion in 150 of 162 patients.

\section{Discussion}

The anesthetic management of patients for microvascular free transfer surgery requires a sound knowledge of circulatory physiology.
It is reasonable to assume that longer periods of surgery are required in more complicated surgical conditions, with meticulous surgical procedures, or with poor wound conditions because it takes more time to achieve anastomosis of blood vessels. In addition to the surgical techniques, there are many factors that affect flap survival. ${ }^{5}$

\section{Temperature Control}

Because of the prolonged surgery and sometimes extensive exposure, it can be difficult to achieve temperature control but it is the most important aspect. Though hypothermia has been shown to be effective in temporary storage of free flaps and severed extremities, the effects of hypothermia like rise in viscosity, vasoconstriction, platelet aggregation, etc. reduce flow in the free flap. We recommend maintaining the patient's body temperature with warming blankets pre- and intraoperatively, and for the first 24-48 hours post surgery. Ambient temperature in the operating room should be relatively high: $24-25^{\circ} \mathrm{C}$ and the temperature differential between skin and core should be kept to less than $2^{\circ} \mathrm{C} .3$

\section{Glucose Control}

From available literature regarding albumin leak, acute and chronic hyperglycemia is associated with vascular leakage and thus tissue edema is more likely to be formed. Hence strict glucose control is recommended. ${ }^{3}$

\section{Hydration}

To maintain adequate microcirculatory perfusion of the transplanted tissue, a hyperdynamic circulation with a high cardiac output, peripheral vasodilatation, and a large pulse pressure is required. Both the donor and the recipient sites have insensible fluid losses and blood losses. Mild-to-moderate hypervolemia reduces the sympathetic vascular tone and dilates the supply vessels to the flap. Though the oxygen delivery to the tissues is probably optimal at a hematocrit level of around 30, most patients can tolerate a drop in hematocrit up to $18-20 \%$, targeting the central venous values of 10-15 cm $\mathrm{H}_{2} \mathrm{O}$ or $3-5 \mathrm{~cm} \mathrm{H}_{2} \mathrm{O}$ above baseline. ${ }^{3}$

\section{Control of Blood Pressure}

During major tumor resection, controlled hypotension is required to improve the surgical field and reduce the blood loss. Other measures which include are (I) positioning to improve the venous drainage and (II) local anesthetic infiltration. Blood pressure can be controlled by various anesthetic techniques like combination of inhalational agent (sevoflurane/isoflurane) and opioids infusion (fentanyl) \pm NTG infusion \pm propofol/dexmetomidine. Mean arterial blood pressure should be maintained at a normal or slightly elevated level during harvesting and anastomosis of the flap to ensure an adequate perfusion pressure through the graft tissue bed. Hypotension is usually secondary to blood loss or vasodilatation and should be treated with fluid replacement. ${ }^{3}$

\section{Microvascular Circulation}

Both crystalloid and colloid infusions are used. We prefer Dextran as it has been shown to be more efficient plasma substitute than both the large volumes of colloids and crystalloids and dextran appears to have beneficial effects on the microcirculation. It has an antithrombotic effect through a reduction in platelet adhesiveness. It also causes a depression of factor VIII activity. Thus, dextran has an advantage in terms of thromboprophylaxis but the amount that can be given has to be limited during major blood loss. Hypertonic saline solutions have been the subject of recent interest. It appears to be an effective plasma volume expander and has beneficial 
cardiovascular effects but due to lack of available literature we prefer Dextran. ${ }^{7}$ Our policy in adults is to infuse Dextran-40 in doses of $1-2 \mathrm{~mL} / \mathrm{kg} /$ hour from the moment the anastomosis is established till 24-36 hours postoperatively. Extensive blood loss can occur during a prolonged procedure and may be covert (e.g., latissimus dorsi donor site). Packed cells to be transfused only if the hemoglobin level falls below $8 \mathrm{~g} / \mathrm{dL}$ or hematocrit less than $18-20 \% .^{3}$

\section{Urine Output}

A urine output of 1-2 $\mathrm{mL} / \mathrm{kg} /$ hour should be maintained intraoperatively and postoperatively with appropriate fluid management. Diuretics are best avoided in these operations because volume depletion compromises flap survival. ${ }^{3}$

\section{Acid-Base Balance and Serum Electrolytes}

As these procedures are of prolonged duration, it mandates blood gas analysis at preset intervals and corrections to be done accordingly. In our institute, we do blood gas analysis every 3-4 hourly. Hypoxia and hypercarbia inadvertently affect microcirculation and thus, graft survival.

\section{Analgesia}

We use intravenous opioids, regional block, or a combination of these two. NSAIDs are usually avoided in the acute perioperative period because of the risk of bleeding and flap hematoma formation.

Regional anesthesia usually consists of a brachial plexus block for upper limb or lumbar epidural for lower limb flaps. Advantages of regional analgesia include excellent analgesia and vasodilatation with improved flap blood flow during and after the operation, reduction in the stress response to surgery and anesthesia, decreased blood loss, reduced incidence of deep vein thrombosis, a hastened postoperative recovery and can prevent vasospasm after replantation. However, there are some major concerns regarding the regional anesthesia, especially epidural anesthesia in microvascular free tissue transfer. As the flap is denervated, sympathectomy produced by an epidural may decrease microcirculatory blood flow in free flaps by decreasing MAP (and hence perfusion pressure) and by diverting the blood away from the flap to normal intact tissues ('steal' phenomenon). This was particularly a concern in hypovolemic patients. We used the epidural towards the end of the case when the blood pressure was satisfactory and the cardiovascular system was adequately filled. We used small increments of $0.125 \%$ or $0.25 \%$ bupivacaine followed by an infusion of $0.15 \%$ bupivacaine with or without fentanyl $(2 \mu \mathrm{g} / \mathrm{mL})$.

\section{Anticoagulation}

The use of various anticoagulants and volume expanders is employed to reduce the probability of vascular thrombosis. Some have been shown to produce an advantage in experimental models, and also potential clinical benefit in retrospective analysis. However, because the rate of thrombosis is quite low with good techniques, detection of a difference between groups is difficult. Despite considerable research on thrombosis in, e.g., cardiovascular disease, antithrombotic regimens in microvascular surgery are generally empiric and few randomized blinded controlled studies exist. The three most commonly used substances include heparin, aspirin, and low-molecular-weight Dextran (also a volume expander).

\section{Heparin}

Heparin is used in microvascular surgery both systemically and topically in irrigation solutions. ${ }^{2,4}$ Though due to limited literature we have not used unfractionated heparin, we prefer Enoxaparin $0.4 \mathrm{~mL}$ subcutaneously.

\section{Aspirin}

Low-dose aspirin is usually recommended for prophylaxis in microvascular free flap surgery. ${ }^{2,4}$

\section{Conclusion}

Due to paucity of literature, most of our general principles in the management of microvascular surgeries are more or less consensus-driven. Perhaps, there is a need for more comprehensive and well-controlled studies as well as formulated guidelines.

Anesthesia for free flaps has some unique problems and challenges, with many uncertainties regarding what constitutes good practice. It is also an exciting and expanding field with great promise in improving quality of life for many. To reduce heat loss, the theater should be prewarmed with the temperature maintained at approximately $26^{\circ} \mathrm{C}$, and the forced air convective warmer with disposable upper or lower body blanket are used.

All solutions to be infused should be heated in a blood warmer. The inspired gases should be heated and moistened by a condenser humidifier or a heat-moisture exchanger which not only helps to prevent body heat loss but also maintains bronchial ciliary activity. All areas of the patient not required to be exposed for surgery are covered to conserve heat.

Adequate postoperative analgesia is important. It reduces sympathetic activity due to pain, thus avoiding hypertension, which could cause bleeding or tissue hypoxia, and allows the patient to cough efficiently and cooperate with physiotherapy.

Hematocrit should be maintained above $30 \%$.

Prophylaxis against deep venous thrombosis is necessary for all patients. Subcutaneous heparin or low-molecular-weight heparin is given preoperatively, while anti-embolism (TED) stockings are used intraoperatively.

\section{References}

1. Quinan J. Anesthesia for Reconstructive Free Flap Surgery. Anaesth Intensive Care Med 2003; 87-90.

2. Hagau $N$, Longrois $D$. Anesthesia for free vascularized tissue transfer. Microsurgery 2009;29(2):161-167. DOI: 10.1002/micr.20584.

3. Khalil AA, Hall JC. Reperfusion injury. Plast Reconstr Surg 2006;117(3):1021-1033. DOI: 10.1097/01.prs.0000204766.17127.54.

4. Hanasono MM, Butler CE. Prevention and Treatment of Thrombosis in Microvascular Surgery. J Reconstr Microsurg 2008;24(5):305-314. DOI: 10.1055/s-2008-1080530.

5. Adams J, Charlton P. British Journal of Anesthesia. CEPD Reviews 2003;3(2):33-37.

6. Sigurdsson $\mathrm{GH}$, Thomson D. Anesthesia and microvascular surgery: clinical practice and research. Eur J Anaesthesiol 1995;12(2): 101-122.

7. Macdonald DJ. Anaesthesia for microvascular surgery: A Physiological Approach. Br J Anaesth 1985;57(9):904-912. DOI: 10.1093/bja/ 57.9.904.

8. Siggurdsson GH. Perioperative fluid management in microvascular surgery. J Reconstr Microsurg 1995;11(1):57-65. DOI: 10.1055/s-20071006512. 